Revue de droit comparé du travail et de la sécurité sociale

3 | 2021

Droits du travail et systèmes nationaux de protection sociale au prisme de la crise sanitaire. Adaptations ou changements profonds?

\title{
Versements exceptionnels à la population suite à la pandémie : une mesure politique ou un dispositif précurseur d'un revenu universel de base?
}

\section{Anna Aleksandrova}

\section{OpenEdition}

Journals

Édition électronique

URL : https://journals.openedition.org/rdctss/2217

DOI : $10.4000 /$ rdctss. 2217

ISSN : 2262-9815

Éditeur

Centre de droit comparé du travail et de la sécurité sociale

Édition imprimée

Date de publication : 1 novembre 2021

Pagination : 204-207

ISSN : 2117-4350

Référence électronique

Anna Aleksandrova, « Versements exceptionnels à la population suite à la pandémie : une mesure politique ou un dispositif précurseur d'un revenu universel de base ? », Revue de droit comparé du travail et de la sécurité sociale [En ligne], 3 | 2021, mis en ligne le 01 novembre 2021, consulté le 18 novembre 2022. URL : http://journals.openedition.org/rdctss/2217 ; DOI : https://doi.org/10.4000/rdctss.2217

Creative Commons -

- CC BY-NC-ND 4.0

https://creativecommons.org/licenses/by-nc-nd/4.0/ 


\section{ANNA ALEKSANDROVA}

UNIVERSITÉ d'ÉTAT DE PENZA

\section{VERSEMENTS EXCEPTIONNELS À LA POPULATION SUITE À LA PANDÉMIE : UNE MESURE POLITIQUE OU UN DISPOSITIF PRÉCURSEUR D'UN REVENU UNIVERSEL DE BASE ?*}

La pandémie de coronavirus a considérablement affecté notre vie, y compris en matière de droits. Beaucoup de lois et de règlements ont été adoptés dans le domaine du travail et de la sécurité sociale. Nombre d'entre eux étaient de nature provisoire, visant à protéger les travailleurs se trouvant dans une situation de chômage forcé. Cependant, certains changements ont été provoqués par des transformations profondes des relations sociales et économiques; ils expriment des tendances importantes dans le développement du droit.

Cet article s'intéressera aux modifications du droit de la sécurité sociale causées par la pandémie en prenant l'exemple des versements exceptionnels à la population (I), puis en analyse leur nature juridique, socio-économique et politique (II).

\section{I - DES VERSEMENTS EXCEPTIONNELS POUR CERTAINES CATÉGORIES DE LA POPULATION}

En 2020-2021, la plupart des pays ont pris des mesures considérables pour soutenir la population se trouvant en situation de confinement; les restrictions apportées à la liberté de mouvement, du travail, etc., empêchant les individus de gagner leur vie et de soutenir leurs familles. L'une de ces mesures fut un soutien financier forfaitaire direct pour les citoyens'. En Russie, la distribution de l'argent à la population (Helicopter money) n'est pas une pratique courante, le gouvernement lui préférant des mesures d'aide ciblées au profit de certaines catégories de la population, à l'instar des entrepreneurs, des chômeurs, des enfants, etc.

Pour les deux premières catégories, le soutien financier était conditionné par un certain nombre de critères : par exemple, les entreprises devaient appartenir aux branches d'industries "les plus touchées par la pandemie "² ; les chômeurs devaient avoir perdu leur emploi pendant la période de restrictions, etc. S'agissant des enfants, le versement de l'allocation familiale exceptionnelle était conditionné au fait d'avoir un enfant d'un certain âge, ce qui semble rapprocher la nature juridique de cette allocation de celle du revenu universel de base.

À l'appui de cette position, plusieurs considérations peuvent être présentées.

* Cette étude a été financée par RFBR, numéro de projet 20-011-00252.

1 Par exemple, au Japon : voir Y. Sekine, «Actualités juridiques internationales. Japon », Revue de droit comparé du travail et de la sécurité sociale, 2021/1, p. 175.

2 https://www.economy.gov.ru/material/dokumenty/perechen otrasley ekonomiki postradavshih $\checkmark$ ezultate rasprostraneniya koronavirusnoy infekcii.html 
Ainsi, il n'existe pas à ce jour, en Russie, d'allocation familiale versée sans conditions aux personnes ayant un enfant de plus de 18 mois (sans conditions signifiant indépendamment du revenu ou de l'activité professionnelle du bénéficiaire).

D'autre part, l'indemnité mensuelle de congé parental (jusqu'aux 18 mois de l'enfant) est versée à la personne soignante. Si le bénéficiaire est assuré, cette indemnité est financée par la Caisse d'assurance sociale, si non par le budget de l'État.

Au titre du soutien aux familles des militaires, une allocation mensuelle est versée pour chaque enfant de militaire (jusqu'à ce que l'enfant atteigne l'âge de 3 ans).

Depuis 2007, un programme «capital maternel » est mis en œuvre en Russie 3 mais les sommes ne sont généralement pas versées en espèces. Transféré au plan bancaire pour financer des dépenses strictement définies par l'État (éducation, intégration sociale des enfants handicapés, etc.), la procédure d'utilisation de ce « capital » est réglementée en détails. Initialement, le capital maternel n'était versé que pour le deuxième enfant, ou les suivants. Ce n'est qu'à partir de 2020 que son versement a été initié dès le premier enfant, le gouvernement étant contraint à cette mesure en raison de la situation démographique difficile du pays.

Ces dernières années, la Russie a adopté une législation sur les indemnités mensuelles versées aux familles les plus démunies ayant un enfant de moins de 3 ans ou un enfant âgé de 3 à 7 ans. Au niveau régional, des allocations sont versées aux familles pauvres ayant un enfant de moins de 16 ans, ainsi que des allocations pour les familles nombreuses. Mais, ces allocations sont attribuées sous condition de ressources, et ne peuvent donc pas être versées à tous les citoyens ayant des enfants.

C'est pourquoi lorsqu'en 2020, le versements en espèces à tous les citoyens ayant des enfants a constitué un immense pas en avant du point de vue de l'action sociale publique. Un décret du Président du 7 avril 20204 prévoyait ainsi versement en avril, mai et juin 2020 de prestations mensuelles octroyées à tous les citoyens ayant des enfants de moins de 3 ans, pour un montant de 5000 roubles par enfant ${ }^{5}$. La démande de prestation devait se faire à distance via le portail des «Services de l'État». Toutefois, en mai 2020, ce décret a été modifié et a introduit une nouvelle prestation avec un versement exceptionnel de 10000 roubles $^{6}$ aux citoyens ayant des enfants âgés de 3 à 16 ans ( versé en été 2020).

La mise en place de ces mesures temporaires de soutien aux familles russes s'inscrit dans la politique sociale générale menée par l'État au moment de la pandémie (des mesures similaires ont été prises par d'autres pays). Ceci ne nous aurait pas incité à les considérer comme une tendance, ou un phénomène nouveau en matière de sécurité sociale, si ces mesures n'avaient pas été répétées par la suite - et ce, plus d'une fois.

3 E. Serebryakova, "Actualité juridique internationale - Fédération de Russie », Revue de droit comparé du travail et de la sécurité sociale », n²018/1, p.157.

4 Décret du Président de la Fédération de Russie du 7 avril 2020 N 249 : http://publication.pravo. gov.ru/ Document/View/0001202004070063

5 Environ 58 euros.

6 Environ 120 euros. 
Ainsi, lors d'une conférence de presse le 17 décembre 20207, le Chef de l'État s'est engagé à offrir un « petit cadeau modeste » pour la nouvelle année à tous les enfants âgés de 0 à 7 ans. Le même jour, un décret a été signé ${ }^{8}$ officialisant un versement exceptionnel de 5000 roubles pour ces catégories d'enfants. En outre, en mai 2021, dans son message annuel au Parlement, le Président a annoncé la mise en place d'un autre versement visant à aider les familles à préparer la rentrée scolaire de leurs enfants. Le décret présidentiel du 2 juillet $2021^{9}$ a ainsi prévu le versement de 10000 roubles pour chaque enfant âgé de 6 à 18 ans, ainsi que pour les étudiants handicapés âgés de 18 à 23 ans. Début septembre 2021, la plupart des bénéficiaires avaient reçu cette somme.

Le Chef de l' État ne s'est pas limité à soutenir les familles avec enfants. Fin août 2021, il a adopté un décret ${ }^{10}$ portant versement d'une somme forfaitaire de 10000 roubles aux retraités (citoyens bénéficiaires de pensions au 31 août 2021 résidant en Russie). Puis, le 30 août 2021, des décrets ont été adoptés pour des versements exceptionnels d'un montant de 15000 roubles aux militaires ${ }^{11}$ et à certaines catégories de fonctionnaires (procureurs, policiers, fonctionnaires du système pénal, etc. $)^{12}$.

\section{II - LA NATURE JURIDIQUE, SOCIO-ÉCONOMIQUE ET POLITIQUE DES VERSEMENTS FORFAITAIRES}

Tous ceux qui connaissent la situation politique en Russie pourraient expliquer l'adoption de ces derniers textes par l'approche des élections législatives (prévues le 19 septembre 2021) et la volonté du Chef de l'Etat d'apporter au parti au pouvoir le soutien non seulement des familles, mais également des retraités, militaires et fonctionnaires. II en est peut-être ainsi. Mais si nous analysons les actes juridiques adoptés en 2020-2021 concernant les versements exceptionnels octroyés à certaines catégories de citoyens, et aux familles principalement, il est possible d'en tirer d'autres conclusions.

Tout d'abord, ces actes ont été adoptés par le Chef de l'État par la voie réglementaire, ce qui implique une procédure simplifiée, par opposition à la procédure législative, complexe et longue. Cette procédure permet de prendre rapidement des décisions administratives et financières, ce qui s'est avéré particulièrement utile pendant la pandémie.

Ensuite, l'article 39 II de la Constitution de la Russie stipule que «les pensions d'État et les prestations sociales sont établies par la loi ». L'interprétation littérale de cette disposition suppose que l'introduction de nouvelles prestations ne peut se faire que par l'adoption

7 http://www.kremlin.ru/events/president/news/64671

8 Décret du Président de la Fédération de Russie du 17 décembre 2020 N 797 : http://publication. pravo. gov.ru/Document/View/0001202012170034

9 Décret du Président de la Fédération de Russie du 2 juillet 2021 N 396 : http://publication.pravo. gov.ru/Document/View/0001202107020040?index $=0 \&$ rangeSize $=1$

10 Décret du Président de la Fédération de Russie du 24 août 2021 N 486 : http://publication.pravo. gov. ru/Document/View/0001202108240001

11 Décret du Président de la Fédération de Russie du 30 août 2021 N 502 : http://www.kremlin.ru/ events/president/news/66530

12 Décret du Président de la Fédération de Russie du 30 août 2021 N 503 : http://publication.pravo. gov. ru/Document/View/0001202108310001 
d'une loi $^{13}$. Selon l'article 104 III de la Constitution, "les projets de loi prévoyant des dépenses du budget fédéral ne peuvent être introduits qu'apres un avis du Gouvernement de la Fédération de la Russie ». Ainsi, l'introduction de nouvelles prestations sociales ne devrait être effectuée que par le Parlement, avec un avis positif du Gouvernement.

On peut constater qu'au cours des années 2020-2021, le Présidenta assezfacilement pris des décisions portant sur la mise en place de nouvelles prestations sociales, en ordonnant à son gouvernement de trouver des ressources à ces fins. Dans les actes reglementaires, ces mesures de soutien sont appelées "versements », et non pas " prestations ", afin que semble-t-il que les décrets présidentiels n'entrent pas en conflit avec la Constitution.

Au-delà de la question de trouver les ressources nécessaires pour financer ces mesures de soutien, la nature discrétionnaire de ces versements interroge en ce qu'ils dépendent uniquement de la volonté du Chef de l'État qui décide de leur nécessité, les annonce, les élabore sous forme de décret, et en contrôle le processus d'octroi. Ceci nous rappelle les mesures de soutien social dont ont bénéficié certaines catégories de la population, à l'époque préindustrielle, et qui étaient réalisées sur décision du monarque. II faut cependant garder à l'esprit que ces versements ont un caractère exceptionnel et irrégulier, et ne peuvent être considérés comme une expression de la politique de l'État dans le domaine de la protection sociale.

Plus généralement, l'introduction de telles mesures témoigne de l'impossibilité pour l'assurance sociale de répondre à tous les défis modernes, y compris la pandémie. Quant à la protection sociale de la famille, l'utilisation des mécanismes assurantiels dans ce champ a toujours été très limitée. En particulier, en Russie, les prestations d'assurance consistent en une allocation aux femmes enceintes, une indemnité mensuelle de congé parental et une allocation de garde d'enfant malade.

Depuis les années 2000, l'État russe a délégué aux pouvoirs régionaux la responsabilité de soutenir les familles avec enfants. L'État a supprimé l'allocation fédérale pour un enfant de moins de 16 ans, qui était versée sans condition de ressources. Actuellement, dans les régions, il n'y a plus que de maigres allocations familiales versées aux plus défavorisés, incomparables avec le minimum vital.

L'introduction des allocations familiales, sous condition de ressources, au niveau fédéral, semble basée sur les principes dépassés du ciblage et de l'évaluation des ressources. II est impossible de renverser la situation démographique défavorable avec de telles méthodes. De plus, dans le monde moderne, le dispositif d'un revenu universel de base devient de plus en plus populaire (la pandémie ayant contribué à l'introduction de mesures similaires dans de nombreux pays). Certains experts et fonctionnaires d'État russes $^{14}$ estiment nécessaire d'instaurer un revenu universel de base qu'elle qu'en soit la forme.

Ainsi, les versements directs à la population, pratiqués de plus en plus fréquemment par la Russie, témoignent de la pénétration progressive des principes du revenu universel de base dans la politique sociale de notre État.

13 Rappelons que la loi est un acte parlementaire, adopté en trois lectures et signé par le Chef de l'Etat.

14 Notamment le Président de la Cour constitutionnelle de la Russie V. Zorkin, le Médiateur (Ombudsman) financier de la Russie Y. Voronin, I'ancien Premier ministre D. Medvedev, etc. 